\title{
Wetland Flora of Gorewada International Biopark, Nagpur, India
}

\author{
Rahul B. Kamble ${ }^{1, *}$, Sandeep G. Hate ${ }^{2}$, Alka Chaturvedi ${ }^{3}$ \\ ${ }^{1}$ Department of Botany, Dr. Ambedkar College, Deekshabhoomi, Nagpur, India \\ ${ }^{2}$ Department of Botany, D.R.B. Sindhu Mahavidyalaya, Nagpur, India \\ ${ }^{3}$ P. G. Department of Botany, University Campus, RTM Nagpur University, Nagpur, India
}

Received June 13, 2020; Revised October 23, 2020; Accepted December 3, 2020

\section{Cite This Paper in the following Citation Styles}

(a): [1] Rahul B. Kamble, Sandeep G. Hate, Alka Chaturvedi , "Wetland Flora of Gorewada International Biopark, Nagpur, India," Advances in Zoology and Botany, Vol. 9, No. 1, pp. 28 - 36, 2021. DOI: 10.13189/azb.2021.090105.

(b): Rahul B. Kamble, Sandeep G. Hate, Alka Chaturvedi (2021). Wetland Flora of Gorewada International Biopark, Nagpur, India. Advances in Zoology and Botany, 9(1), 28 - 36. DOI: 10.13189/azb.2021.090105.

Copyright $\subseteq 2021$ by authors, all rights reserved. Authors agree that this article remains permanently open access under the terms of the Creative Commons Attribution License 4.0 International License

\begin{abstract}
Gorewada reservoir and adjacent area (1885 Ha) now converted into International Biopark abodes a variety of plant community to its credit. A study has been carried out to elucidate the aquatic flora with diverse plant wealth, indicator plants and their ecological status in wetlands of Gorewada catchment area.114 plants species with 33 families have been identified from the Gorewada wet land area. 67 species belong to dicotyledons and 47 are monocotyledons.
\end{abstract}

Keywords Gorewada International Biopark, Nagpur, Wetland Flora, Indicator Categories

\section{Introduction}

Biodiversity accomplishes all vital requirements of organisms to complete their life cycle and amongst humans indirectly or directly dependent on both terrestrial as well as aquatic biodiversity, as both biodiversities provides natural resources in all ways to humans like cultural, economic, aesthetic, educational and scientific etc. Biodiversity covers all organisms like microorganism, plants and animals along with its all diverse genetic variations in species, varieties and populations in different habitats, ecosystems and in their natural areas, and its groups increases richness in natural environment [1].
Wetlands are vital parts of the hydrological cycle, highly productive, support exceptionally large biological diversity and provide a wide range of ecosystem services, such as food and fibre; waste assimilation; water purification; flood mitigation; erosion control; groundwater recharge; microclimate regulation; enhance aesthetics of the landscape; support many significant recreational, social and cultural activities, besides being a part of our cultural heritage [2].

The composition of a plant community is determined by a complex interaction of several biotic as well as abiotic factors including climate, soil type, position in the landscape and competition between plant species. Amongst which wetlands are the very important aquatic ecosystem, which maintains the balance in environment. Wetlands have seasonal variation in its floristic composition. Consequently, botanical explorations of wetland plants are necessary to gain more knowledge on species richness as well as their geographical distribution. Works on floristic studies of documentation of diversity of aquatic plants and wetlands flora in various water reservoirs and wetland areas of different states of India were carried out by many workers $[3,4,5,6,7,8,9,10]$. Besides these there are several researchers from Maharashtra state and its various districts and regions, explored the aquatic and wet lands plants diversity [11,12,13,14,15,16,17,18,19,20,21, 22]. 


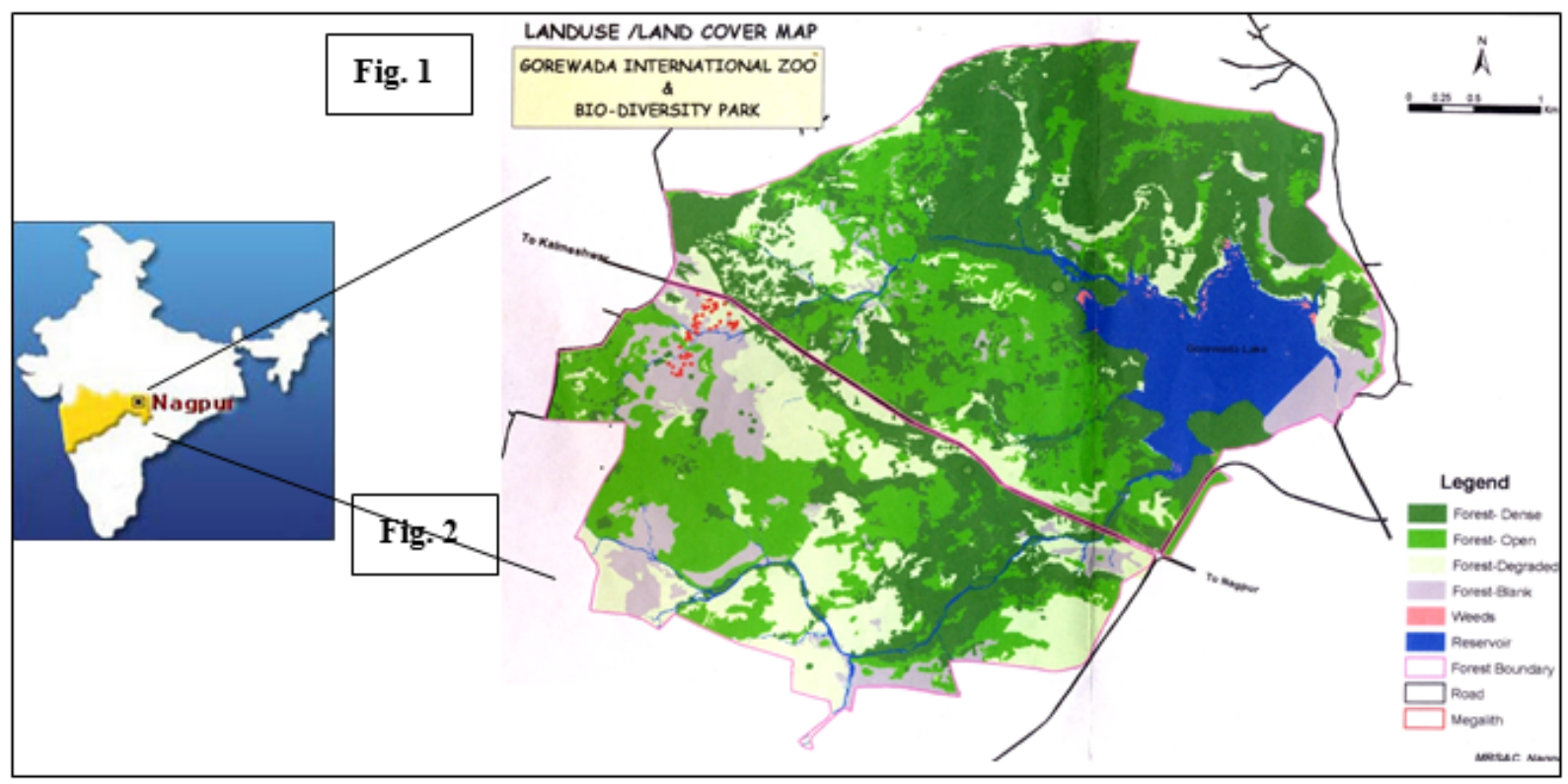

Figure 1. Map of Gorewada International Biopark

Nagpur is one of the greenest cities of India with 18 per cent of its area under forests and plantations, 17 per cent under cultivation and 2 per cent under water bodies [23]. To maintain ecological equilibrium, there is a need of documentation of floristic composition of wetlands, which plays a crucial role as pollution indicator. From this the assessment of conservation of threatened taxa which are associated with wetland habitats also carried out. In view of this fact, the present study is meant to prepare the documentation of the phytodiversity of wetland of Gorewada International Biopark (Proposed), Nagpur, the first exploration of the kind in this area.

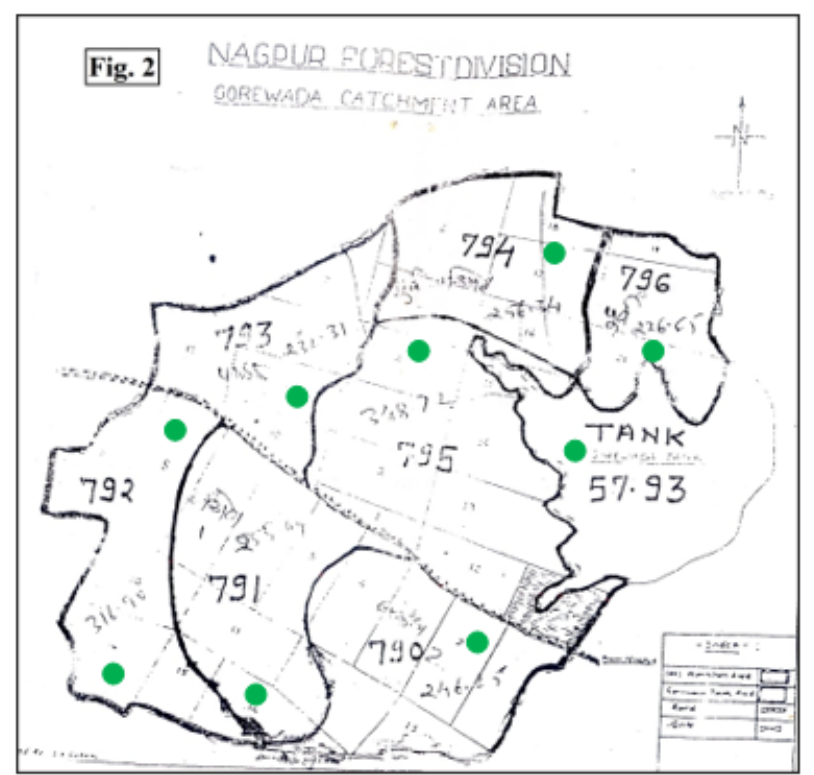

Figure 2. Compartments wise Map showing wetlands

\section{Material and Methods}

\subsection{Study Area}

Proposed Gorewada International Biopark lies between latitudes $20^{\circ} 35^{\prime}$ and $21^{\circ} 44^{\prime}$ North and longitudes $78^{\circ} 15^{\prime}$ and $79^{\circ} 40^{\prime}$ East. It extends over to 1885 ha of reserved forests, in outskirts of Nagpur city. All area is divided into 7 beats (Compartments) viz. 790, 791, 792, 793, 794, 795, 796 and one water catchment area. Land is well drained and form catchment area of Gorewada Lake, known for its scenic beauty. Gorewada water reservoir, an area of around 57.93 sq. Hectare is situated on the eastern side of the proposed zoo and bio-park. Northern bank of the lake is made by beat number 796 and western side by beat number 795. Almost all compartments have well drainage system in the form of ponds and streams towards the water reservoir. The annual average rainfall of the area is $900 \mathrm{~mm}$ and the annual mean temperature varies from $10^{\circ}$ $\mathrm{C}$ minimum to $45^{\circ} \mathrm{C}$ maximum [24].

\subsection{Methods}

For the study of wetland flora, the survey in and around ponds, lakes, streams with extensive field visits have been done in various time of the year to cover all the wetlands of the area in different seasons during 2008 to 2013.The different wetland of each 7 compartments along with water reservoir often visited are shown in map The methodology adopted for the data on taxa distribution within the forest range were collected particularly with the help of two sources- field observations and identification with pertinent literatures [25,26,27].Besides that the information on habit, habitat and flowering was carried out in the both dry and wet seasons. The identification of 
specimens was carried out by consulting relevant literature and regional floras [28,29,30,31] and morpho-ecological classification viz. I) Submerged anchored, II) Free Floating, III) Floating leaved anchored, IV) Floating shoot anchored,V) Emergent anchored and VI) Helophytes [32].

\section{Results and Discussion}

Exhaustive field visits to the all wetland areas in 7 compartments and area adjoining the water reservoir carried out in all seasons of the year and documented all floral components. The statistical analysis of the floristic components also carried out. A total of 114 species from 33 families were identified from the Gorewada wet land area. 67 species belong to dicot and 47 are monocots
(Table No.2). Some of the major dominant wetland phyto-diversity are Hydrilla, Utricularia, Vallisneria, Ceratophyllum, Azolla, Eichhornia, Ipomea, Lemna, Trapa., Nymphoides indica, Spigelia anthelmia L. [33] etc. According to ecological classification, majority of the taxa belong to category V, i.e. muddy elements. Category I, submerged species are represented by Vallisneria, Nechmandra, Naias, Ceratophyllum and Hydrilla, While Ottelia, Aponogeton and Limnophyllum forms the floating leaves category, II. Typha and Ipomea fistulosa are the most frequenttaxa of category IV. (Table. No.1\& Figure. 3 ). besides these, algae, aquatic fungi, bryophytes and pteridophytes are also major parts of the wetland ecosystem. The detailed list of floral components according to its habits and ecological class occurred in Gorewada International Biopark given below. (Table No. 1).

Table 1. .Floristic diversity of wetland belonging to different morpho-ecological classes and plant indicators from the study area are given as below

\begin{tabular}{|c|c|c|c|c|}
\hline Sr. No & Botanical Name & Family & Habit & $\begin{array}{c}\text { Ecological } \\
\text { class }\end{array}$ \\
\hline 1. & Justicia diffusaWilld. & Acanthaceae & Wet Patches & VI \\
\hline 2. & Justicia simplex D.Don. & Acanthaceae & Wet Patches & VI \\
\hline 3. & Rungia pectinata(L.) Nees. & Acanthaceae & Moist Places & VI \\
\hline 4. & Hygrophila auriculata (K. Schum.) Heine & Acanthaceae & Wet Patches & VI \\
\hline 5. & Justicia procumbens L. & Acanthaceae & Margin of lake & VI \\
\hline 6. & Justicia quinquangularis Koen. & Acanthaceae & Margin of lake & VI \\
\hline 7. & Alternanthera sessilis (L.) R.Br. & Amaranthaceae & Wet mud & VI \\
\hline 8. & Celosia argentea $\mathrm{L}$. & Amaranthaceae & Moist Region & VI \\
\hline 9. & Amaranthus viridis L. & Amaranthaceae & Moist Places & VI \\
\hline 10. & Aponogeton natans (L.) Engl. \& Krause & Aponogetonaceae & Submerged & VI \\
\hline 11. & Gnaphalium polycaulon Pers. & Asteraceae & Moist Places & VI \\
\hline 12. & Blumea lacera (Burm.f.) DC. & Asteraceae & Margin of Lake & VI \\
\hline 13. & Caesulia axillaris Roxb. & Asteraceae & Margin of Lake & VI \\
\hline 14. & Cyathocline purpurea (D.Don.) O.Ktze. & Asteraceae & Dry Mud Patches & VI \\
\hline 15. & Glossocardia bosvallea (L.f.) DC. & Asteraceae & Sandy / Moist & VI \\
\hline 16. & Sonchus brachyotus DC. & Asteraceae & Moist Region & VI \\
\hline 17. & Vernonia cinerea (L.) Less & Asteraceae & Moist Region & VI \\
\hline 18. & Trichodesma sedgwickianum Banerjee & Boraginaceae & Margin of Lake & VI \\
\hline 19. & Heliotropium supinum L. & Boraginaceae & Margin of Lake & VI \\
\hline 20. & Ceratophyllum demersum $\mathrm{L}$. & Ceratophyllaceae & Submerged & I \\
\hline 21. & Cleome chelidonii L.f. & Cleomaceae & Wet Patches & VI \\
\hline 22. & Terminalia arjuna (Roxb.) Wt. \&Arn. & Combretaceae & Margin of Lake & VI \\
\hline 23. & Commelina benghalensis L. & Commelinaceae & Wet mud patches & VI \\
\hline 24. & Cyanotis axillaris (L.) D.Don ex Sweet & Commelinaceae & Wet mud patches & VI \\
\hline 25. & Merremia emarginata (Burm.f.) Hall.f. & Convovulaceae & Moist Region & VI \\
\hline 26. & Evolvulus alsinoides (L.) L. & Convovulaceae & Wet Patches & VI \\
\hline 27. & Ipomoea fistulosa Mart. ex Choisy & Convolvulaceae & Wet mud patches & III \\
\hline 28. & Eleocharis atropurpurea (Retz.) K.B. Presl. & Cyperaceae & Wet Patches & VI \\
\hline 29. & Cyperus iria L. & Cyperaceae & Wet Patches & VI \\
\hline 30. & Cyperus corymbosus Rottb. & Cyperaceae & Wet Patches & VI \\
\hline
\end{tabular}


Table 1 Continued

\begin{tabular}{|c|c|c|c|c|}
\hline 31. & Fuirena glomerata Lamk. & Cyperaceae & Dry Mud patches & VI \\
\hline 32. & Kyllinga brevifolia Rottb. & Cyperaceae & Wet Patches & VI \\
\hline 33. & Cyperus compressus L. & Сyperaceae & Moist Region & VI \\
\hline 34. & Cyperus niveus Retz. & Cyperaceae & Moist Region & VI \\
\hline 35. & Fimbristylis aphylla Steud. & Cyperaceae & Moist Region & VI \\
\hline 36. & Fimbristylis dichotoma (L.) Vahl. & Cyperaceae & Moist Region & VI \\
\hline 37. & FImbristylis ferruginea (L.) Vahl. & Cyperaceae & Margin of lake & VI \\
\hline 38. & Fimbristylis spathacea Roth. & Cyperaceae & Margin of lake & VI \\
\hline 39. & Kyllinga nemoralis (Forst.) Dandy & Cyperaceae & Moist Region & VI \\
\hline 40. & Eriocaulon cinereum R.Br. & Eriocauliaceae & Wetland & VI \\
\hline 41. & Eriocaulon quinquangulare L. & Eriocauliaceae & Wetland & VI \\
\hline 42. & Eriocaulon luzulaefolium Mast. & Eriocaulaceae & Wet Patches & VI \\
\hline 43. & Phyllanthus amarus Schum. \&Thonn. & Euphorbiaceae & Dry Mud patches & VI \\
\hline 44. & Euphorbia rosea Retz. & Euphorbiaceae & Dry Mud patches & VI \\
\hline 45. & Euphorbia hirtaL. & Euphorbiaceae & Margin of lake & VI \\
\hline 46. & Chrozophoraprostrata Dalz. & Euphorbiaceae & Dry Mud patches & VI \\
\hline 47. & Crotalaria albida Heyne ex Roth & Fabaceae & Dry Mud patches & VI \\
\hline 48. & Crotalaria sericea Retz. & Fabaceae & Dry Mud patches & VI \\
\hline 49. & Indigofera cordifolia Heyne ex Roth & Fabaceae & Dry Mud patches & VI \\
\hline 50. & Indigofera trifoliata $\mathrm{L}$. & Fabaceae & Dry Mud patches & VI \\
\hline 51. & Smithia conferta J.E. Sm. & Fabaceae & Dry Mud patches & VI \\
\hline 52. & Aeschynomene indica L. & Fabaceae & Wet Patches & VI \\
\hline 53. & Zornia gibbosa Spanoghe & Fabaceae & Dry Mud patches & VI \\
\hline 54. & Zornia diphylla (L.) Pers. & Fabaceae & Dry Mud patches & VI \\
\hline 55. & Sesbania sesban (L.) Merr. & Fabaceae & Wet patches & VI \\
\hline 56. & Alysicarpus vaginalis (L.) DC. & Fabaceae & Wetland & VI \\
\hline 57. & Centaurium centaurioides (Roxb.) Rao \& Hemadri & Gentianaceae & Wetland & VI \\
\hline 58. & Canscora decurrens Dalz. & Gentianaceae & Margin of lake & VI \\
\hline 59. & Exacum pedunculatum L. & Gentianaceae & Margin Region & VI \\
\hline 60. & Hoppea dichotoma Willd. & Gentianaceae & Wetland & VI \\
\hline 61. & Nymphoides cristata (Roxb.) O. Ktze. & Gentianaceae & Surface of Lake & II \\
\hline 62. & Hydrilla verticillata (L.f.) Royle. & Hydrocharitaceae & Submerged & I \\
\hline 63. & Ottelia alismoides (L.) Pers. & Hydrocharitaceae & Submerged & III \\
\hline 64. & Vallisneria spiralis L. & Hydrocharitaceae & Submerged & I \\
\hline 65. & Nechamandra alternifolia (Roxb.) Thw. & Hydrocharitaceae & Submerged & I \\
\hline 66. & Leucas aspera (Willd.) Spreng. & Lamiaceae & Moist Places & VI \\
\hline 67. & Ocimum basilicum L. & Lamiaceae & Margin of Lake & VI \\
\hline 68. & Lemna paucicostata Hagelm & Lemnaceae & Surface of Lake & II \\
\hline 69. & Scilla hyacinthina (Roth.) Macbr. & Liliaceae & Dry Mud patches & VI \\
\hline 70. & Curculigo orchioides Gaertn. & Liliaceae & Moist places & VI \\
\hline 71. & Gloriosa superba L. & Liliaceae & Moist places & VI \\
\hline 72. & Mitreola petiolata (J.F. Gmel.) Torr. \& Gray. & Loganiaceae & Wet Patches & VI \\
\hline 73. & Spigelia anthelmia L. & Loganiaceae & Wet Patches & VI \\
\hline 74. & Rumex dentatus $\mathrm{L}$. & Lythraceae & Margin of Lake & VI \\
\hline 75. & Ammannia multiflora Roxb. & Lythraceae & Dry Mud & VI \\
\hline
\end{tabular}


Table 1 Continued

\begin{tabular}{|c|c|c|c|c|}
\hline 76. & Rotala indica (Willd.) Koehne. & Lythraceae & Wet patches & VI \\
\hline 77. & Woodfordia fruticosa (L.) Kurz. & Lythraceae & Margin of Lake & VI \\
\hline 78. & Najas minor All. & Najadaceae & Submurged & IV \\
\hline 79. & Ludwigia octovalvis (Jacq.) Raven. & Onagraceae & Wet mud & VI \\
\hline 80. & Biophytum sensitivum (L.) DC. & Oxalidaceae & Wet Mud & VI \\
\hline 81. & Aristida funiculata Trin. & Poaceae & Dry Mud patches & VI \\
\hline 82. & Chrysopogon fulvus (Spreng.) Chiov. & Poaceae & Dry Mud patches & VI \\
\hline 83. & Coix lacryma-jobi L. & Poaceae & Wet Patches & $\mathrm{V}$ \\
\hline 84. & Dichanthium aristatum (Poir.) C.E. Hubb. & Poaceae & Wet Patches & VI \\
\hline 85. & Digitaria ciliaris (Retz.) Koeler & Poaceae & Dry Mud patches & VI \\
\hline 86. & Eragrostiella brachyphylla (Stapf.) Bor. & Poaceae & Dry Mud patches & VI \\
\hline 87. & Heteropogon contortus (L.) Pal.-Beauv. & Poaceae & Dry Mud patches & VI \\
\hline 88. & Oplismenus compositus (L.) Pal.-Beauv. & Poaceae & Dry Mud patches & VI \\
\hline 89. & Paspalidium flavidum (Retz.) A. & Poaceae & Wet Patches & VI \\
\hline 90. & Paspalidium geminatum (Forssk.) Stapf. & Poaceae & Wet Patches & VI \\
\hline 91. & Pennisetum pedicellatum Trin. & Poaceae & Dry Mud patches & VI \\
\hline 92. & Panicum miliare Lamk. & Poaceae & Dry Mud patches & $\mathrm{VI}$ \\
\hline 93. & Setaria verticillata (L.) Pal.-Beauv. & Poaceae & Dry Mud patches & VI \\
\hline 94. & Themeda triandra Forssk. & Poaceae & Dry Mud patches & VI \\
\hline 95. & Cynodon dactylon (L.) Pers. & Poaceae & Margin of Lake & $\mathrm{VI}$ \\
\hline 96. & Eragrostis gangetica (Roxb.) Steud. & Poaceae & Margin of Lake & VI \\
\hline 97. & Eragrostis aspera (Jacq.) Nees. & Poaceae & Margin of Lake & VI \\
\hline 98. & Setaria intermedia Roem. \&Schult. & Poaceae & Margin of Lake & VI \\
\hline 99. & Polygala arvensis Willd. & Polygalaceae & Moist Places & VI \\
\hline 100. & Polygonum glabrum Willd. & Polygonaceae & Dry Mud & $\mathrm{V}$ \\
\hline 101. & Potamogeton nodosus Poir. & Potamogetonaceae & Submerged & I \\
\hline 102. & Potentilla supine L. & Rosaceae & Dry Mud patches & VI \\
\hline 103. & Dentella repens (L.) Forst. & Rubiaceae & Moist Places & VI \\
\hline 104. & Oldenlandia corymbosa $\mathrm{L}$. & Rubiaceae & Margin of Lake & VI \\
\hline 105. & Striga densiflora (Benth.) Benth. & Scrophulariaceae & Wet Patches & VI \\
\hline 106. & Sopubia delphiniifolia (L.) G.Don. & Scrophulariaceae & Moist Places & VI \\
\hline 107. & Lindernia crustacea (L.) F.V. Muell. & Scrophulariaceae & Moist Places & VI \\
\hline 108. & Limnophila heterophylla (Roxb.) Benth. & Scrophulariaceae & Wet mud & IV \\
\hline 109. & Buchnera hispida Buch.-Ham. & Scrophulariaceae & Moist Places & VI \\
\hline 110. & Bacopa monnieri (L.) Wettstein & Scrophulariaceae & Wet mud. & $\mathrm{VI}$ \\
\hline 111. & Verbascum chinense (L.) Santapau & Scrophulariaceae & Margin of Lake. & VI \\
\hline 112. & Trapa natans L. & Trapaceae & Surface of Lake & III \\
\hline 113. & Typha angustata Chaubard \& Bory & Typhaceae & Wet mud. & $\mathrm{V}$ \\
\hline 114. & Phyla nodiflora (L.) Greene & Verbenaceae & Moist Places & $\mathrm{VI}$ \\
\hline
\end{tabular}

Note: I) Submerged anchored, II) Free Floating, III) Floating leaved anchored, IV) Floating shoot anchored, V) Emergent anchored and VI) Helophytes

Table 2. Statistical analysis showing percentage of Dicotyledonous and Monocotyledonous families, genera and species

\begin{tabular}{|c|c|c|c|c|}
\hline & \multicolumn{2}{|c|}{ Dicots. } & \multicolumn{2}{c|}{ Monocots. } \\
\hline & No. & No. & 30.30 \\
\hline Families & 23 & 69.70 & 10 & 37.23 \\
\hline Genera & 59 & 62.76 & 35 & 41.22 \\
\hline Species & 67 & 58.77 & 47 & \\
\hline Total & & & 114 & \\
\hline
\end{tabular}




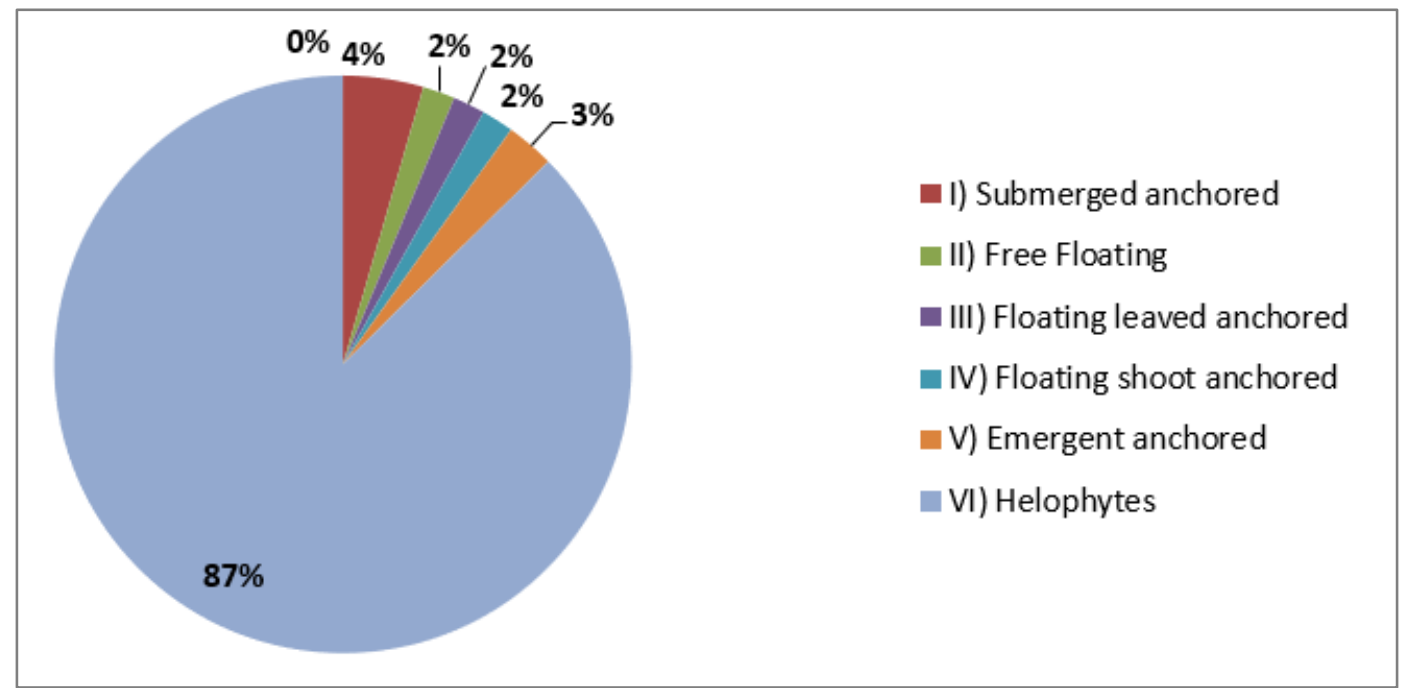

Figure 3. Pi diagram showing the composition of life form of aquatic macrophytes of the wetlands of the study area

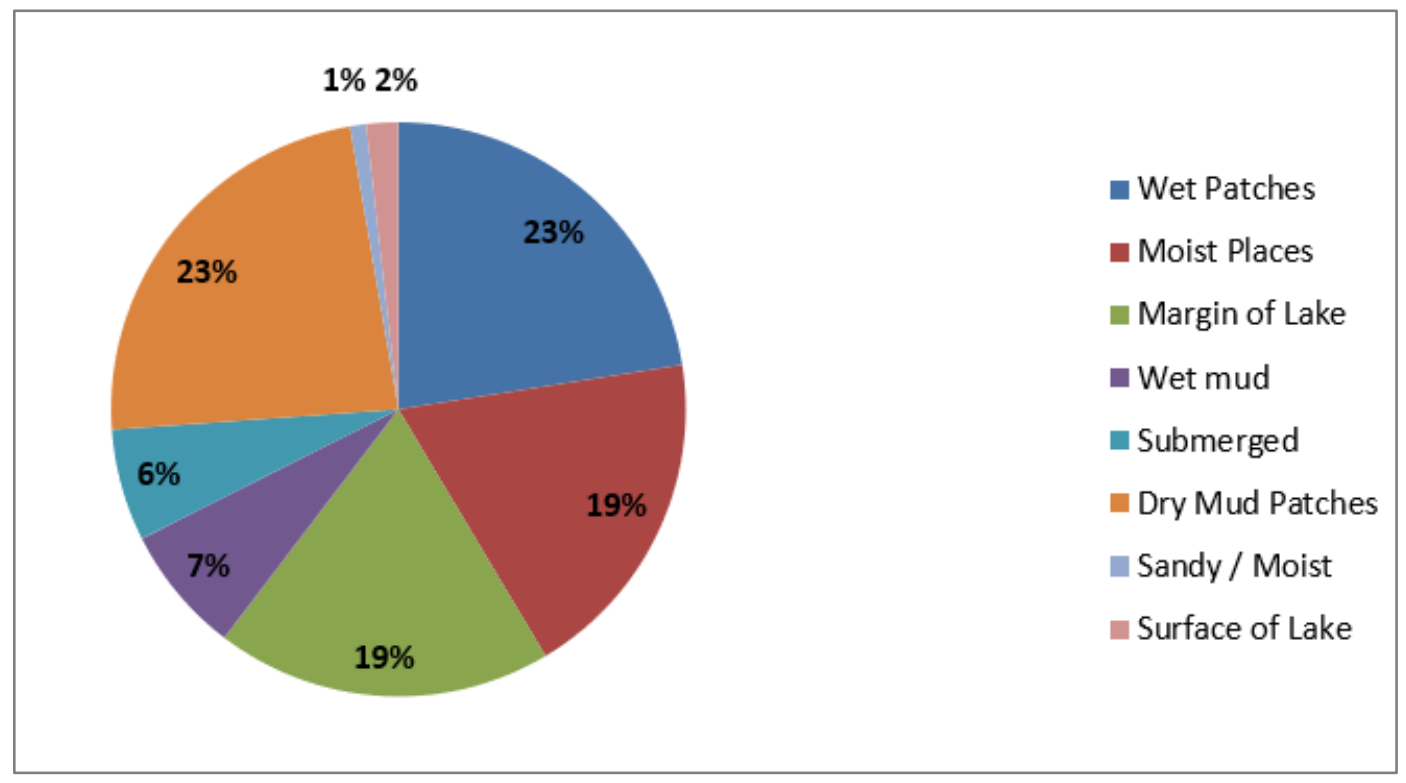

Figure 4. Habit Sharing of flora in Gorewada Wetland 
Table3. List of Dominating Wetland Plants with Indicator Category

\begin{tabular}{|l|c|c|}
\hline Sr. No. & Scientific Names & $\begin{array}{c}\text { Indicator } \\
\text { Category }\end{array}$ \\
\hline 1. & Bacopa monnieri (L.) Wettstein & OBL \\
\hline 2. & Eriocaulon cinereum R.Br. & OBL \\
\hline 3. & Phyla nodiflora (L.) Greene & FACW \\
\hline 4. & Typha angustata Chaubard \& Bory & OBL \\
\hline 5. & Ipomoea fistulosa Mart. ex Choisy & FAC \\
\hline 6. & Vallisneria spiralis L. & OBL \\
\hline 7. & Hydrilla verticillata (L.f.) Royle & OBL \\
\hline 8. & Ottelia alismoides (L.) Pers. & OBL \\
\hline 9. & Najas minor All. & OBL \\
\hline 10. & Phoenix sylvestris (L.) Roxb & UPL \\
\hline 11. & Terminalia arjuna (Roxb.) & FACU \\
\hline 12. & Wponogeton natans (L.) Engl. \& Kr. & OBL \\
\hline 13. & Cleome chelidonii L.f. & OBL \\
\hline 14. & Crotalaria sericea Retz. & FACW \\
\hline 15. & Phyllanthus amarus Schum. \&Thn. & FAC \\
\hline 16. & Nymphoides cristata (Roxb.) O.Ktze & OBL \\
\hline 17. & Lindernia crustacea (L.) F.V. Muell. & OBL \\
\hline 18. & Oldenlandia corymbosa L. & OBL \\
\hline 19. & Coix lacryma-jobi L. & OBL \\
\hline 20. & Smithia conferta J.E. Sm. & UPL \\
\hline 21. & Ludwigia octovalvis (Jacq.) Raven. & FACU \\
\hline & & \\
\hline & & OAr \\
\hline
\end{tabular}

Wetland Indicator Categories based on differences in expected frequency of occurrence of wetland plant species [34], probability of occurrence in wetlands as opposed to upland habitats, with obligate wetland species having a percentage probability of occurrence in wetlands (OBL)> 99\%; facultative wetland species (FACW), 67\% - 99\%; facultative species (FAC), 34\% - 66\%; facultative upland species (FACU), 1\% - 33\%; and obligate upland species (UPL), $<1 \%$. According to this, the dominant wetland plants were categorized. The OBL indicator category comprises Bacopa monnieri (L.) Wettstein, Eriocaulon cinereum R.Br., Typha angustata Chaubard \& Bory, Vallisneria spiralis L., Hydrilla verticillata (L.f.) Royle, Ottelia alismoides (L.) Pers. Najas minor All., Aponogeton natans (L.) Engl. \& Kr., Cleome chelidonii L. f., Nymphoides cristata (Roxb.) O. Ktze, Lindernia crustacea (L.) F.V. Muell., Oldenlandia corymbosa L.,
Coix lacryma-jobi L. Facultative wetland species (FACW) are Phyla nodiflora (L.) Greene and Crotalaria sericea Retz.; facultative species (FAC) with Ipomoea fistulosa Mart. ex Choisy and Phyllanthus amarus Schum. \&Thn.; facultative upland species (FACU) are Terminalia arjuna (Roxb.) Wt.\&Arn. and Ludwigia octovalvis (Jacq.) Raven. and obligate upland species (UPL) represented with Phoenix sylvestris (L.) Roxb. and Smithia conferta J.E. Sm. (Table.3).

Gorewada Lake is one of the important water reservoirs of the Nagpur, from where supply of the drinking water to the different parts of the city takes place. At the same time its expanded marshy land provides suitable habitats for breeding of number of water animals. Large population of macrophytes of wetlands is provider of not only habitat but also the much needed food for number of organisms.

\section{Conclusions}

Wetlands have a great diversity of plants needing wetness of diverging extents. These plants are accordingly adapted to the local availability of water and many show transitions between annual and perennial forms and also their type of growth forms. Weed, a controversial class of plants, its invasion is a major problem in wetlands resulting in increased competition for resources such as water, light, nutrients and space [34]. Wetland plants can be threatened by changes in both surface water and groundwater levels as a result of human activities. Wetland plants are sensitive to changes in hydrology which result in a wetland having too much or too little water. The anthropogenic activities like deforestation; overgrazing and developmental activities etc. are causative factors for the acceleration of soil erosion and consequent siltation of wetlands. For successful implementation of conservation, involvement of people and nongovernmental organizations is crucial. People can be involved in de-siltation of lake, afforestation in the catchment area and generating awareness

\section{Acknowledgements}

Authors are thankful to the Director, Gorewada Projects, Forest Department, Govt. of Maharashtra and Local peoples besides the forest of Gorewada. 


\section{Appendix}

\section{Plate No 1:- Wetland patches}

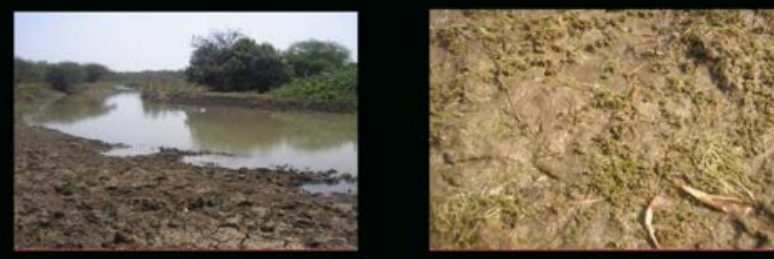

\section{Compartment No 794 Compartment No 796}
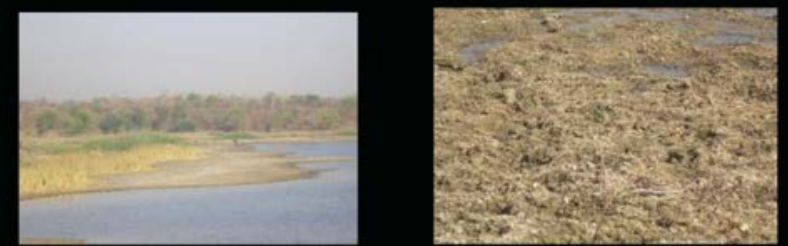

Compartment No 795 Compartment No 795
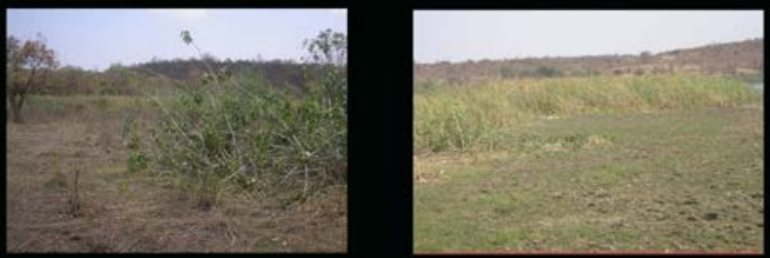

Compartment No 794 Compartment No 796
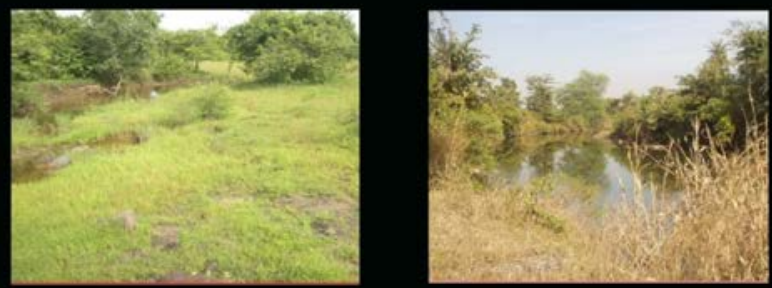

Compartment No 795 Compartment No 790

\section{REFERENCES}

[1] Kulshrestha SK. Biodiversity conservation of Freshwater ecosystem in India.EnviroNews,11(2). 2005

[2] Ramachandra T.V. Wetlands: Need for Appropriate Strategies for Conservation and Sustainable Management. Journal of Basic and Applied Biology, 4(3), 1-17, 2010.

[3] Agharkar, S. P. The present position of our knowledge of the aquatic flora of India. Indian. Bot Soc. 3 : 252-260. 1923.

[4] Biswas, K. and Calder, C.C. Handbook of Common Water and Marsh Plants of India and Burma. New Delhi. 1936.

[5] Bhandari, B. B., Singh, B. and Desai, B. L. Water Plants. New Delhi. 1962.

[6] Subramanyam, K. Aquatic Angiosperms. New Delhi. 1962.

[7] Cook, C. D. K. Aquatic and Wetland Plants of India, New York. 1996.

[8] Bandyopadhyay S. and Mukherjee S.K. Diversity of Aquatic and Wetland Vascular Plants of Koch Bihar District, West Bengal. Plant Taxonomy: Advances and Relevance, Pages 223-244 Eds. A.K. Pandey, Jun Wen \& J.V.V. Dogra. CBS Publ \& Distributors, New Delhi, 2005.

[9] Mitra S. and Mukherjee S.K. Diversity of Aquatic and Wetland Plants of West Dinajpur District, West Bengal. In book: Biodiversity- Impact and Assessment. (pp.169 - 184) Publisher: P. C. Trivedi (ed.), Biodiversity- Impact and Assessment. Pointer Publishers, Jaipur, PP. Editors: P. C. Trivedi. 2009.

[10] Sukumaran S. and Jeeva S. Angiosperm flora from wetlands of Kanyakumari district, Tamilnadu, India. Check List 7(4), pp 486-495. 2011 
[11] Dhore Mukund Manohar and Lachure Paresh Shyaam. Survey of Aquatic Macrophyte diversity in Yavatmal District, Maharashtra, India., Int. J. of Life Sciences, 2(3): 273-275. 2014.

[12] Wainganga Water Partnership. Report on Wetland Management Plan for 5 villages of Bhandara and Gondia districts of Maharashtra having catchment area of Wainganga River. India Water Partnership. 2017.

[13] Kshirsagar Ayodhya D. and Venkat R Gunale, Diversity of aquatic macrophytes from River Mula Pune City, MS, India. Sci. Res. Rept, 3(1):09-14. 2013.

[14] Idhole R.L., Chhaba S. G. and Dabhade D. S. Biodiversity of Aquatic Weeds in Washim Region of Maharashtra, India. Indian Streams Research Journal, Vol 6 (5): 1-5. 2016.

[15] Deshmukh UB, MB Shende, OS Rathor. Aquatic macrophytes biodiversity assessment from Asolamendha reservoir of Chandrapur district, Maharashtra State (India). IJAR 2016; 2(1): 293-298.

[16] Rohankar, L., Telkhade, P., Dahegaonkar, N. and Lonkar, A. Macrophyte diversity in rural lake, Aheri, dist. Gadchiroli, Maharashtra state, India. Bionano Frontier. 5(2): 203-204. 2012.

[17] Purshuramkar B.B., Telkhade P.M., \& Khune C.J. Macrophyte diversity of Chulbandh reservoir, Murdoli, district Gondia, Maharashtra, India. Online International Interdisciplinary Research Journal. 3(2): 216-218. 2013.

[18] Tiwari V.J. Biodiversity of aquatic plants of Shivnibandh lake of Sakoli tehsil of Bhandara district of MS, India. Int. J. of Life Sciences. A2: 211-213. 2014.

[19] Harney, N.V. Macrophytes biodiversity of Dudhala lake of Bhadrawati, district- Chandrapur (MS), India. Asian Journal of Multidisciplinary Studies. 2(4): 69-72. 2014.

[20] Reddy B. Mallesh and A. Chaturvedi. Study of Aquatic and Associated Macrophytes from the Major Rivers of the Chandrapur District, Maharashtra. International Journal of Science, Environment and Technology, Vol. 5, No 6, 3774 3782. 2016.

[21] Dani P.A, M.B. Wadekar, S.D Narkhede. Monocotyledonous plant diversity of wetlands in kurkheda taluka, District Gadchiroli, Maharashtra. International Journal of Academic Research and Development, 5(1); 01-05, 2020.

[22] Dhore, Manik; Dhore, Mukund; Dabhadkar, Dinesh; Zade, Varsha. Survey of Macrophyte Diversity in Certain Wetland of Akola district, Maharashtra, India. Golden Research
Thoughts; 1(8), p83, 2012.

[23] Chaturvedi A, Kamble R.B., Patil NG \& Chaturvedi A City-Forest Relationship In Nagpur, One Of The Greenest City Of India. Urban Forestry \& Urban Greening Elsevier, 12(1): 79-87. 2013.

[24] Rahul B. Kamble, Sandeep Hate, Arvind Mungole, \& Alka Chaturvedi. A Note On Floristic Diversity And Anthropogenic Impacts In Gorewada International Bio-Park (Proposed), Nagpur (Maharashtra), In Biodiversity Assessment and Conservation: Edited by Maiti\& Mukherjee, Vol 1: Pp: 396-402.2012.

[25] Brock, M.A. Are there seeds in your wetland? Assessing wetland vegetation, LWRRDC, Canberra and UNE, Armidale. 1997.

[26] Barbour, M.G., Burk, J.H. and Pitts, W.D. Terrestrial Plant Ecology, Second Edition, The Benjamin/Cummings Publishing Company, Inc., California. 1987.

[27] Mueller-Dombois, D. and Ellenberg, H. Aims and Methods of Vegetation Ecology. John Wiley and Sons, Sydney. 1974.

[28] Ugemuge N R. Flora of Nagpur District; Shree Publication, Nagpur.1986.

[29] Sharma B.D., Kartikeyan S. \& Singh N.P., Flora of Maharashtra State (Monocotyledones) Botanical Survey of India. 1990

[30] Singh N.P. \& Kartikeyan S., Flora of Maharashtra State (Dicotyledones) Vol. I Botanical Survey of India2000.

[31] Singh, N.P., Lakshminarsimhan P., Kartikeyan S., \& Prasanna P.V., Flora of Maharashtra State (Dicotyledones) Vol. II Botanical Survey of India.2001.

[32] Daubenmire, R.F. Plants and Environment: A Textbook of Plant Autecology. New York: Jonn Wiley and Sons 148 p.1947.

[33] Kamble Rahul B and Chaturvedi Alka. Spigelia anthelmia L. (Spigeliaceae) A New Record from Eastern Maharashtra. Bioinfolet, 7: 306-308.2010.

[34] Reed, P. B. Jr. National list of plant species that occur in wetlands: National summary. Biological Report 88. U.S. Fish and Wildlife Service, Washington, D.C., USA. Website http://www.fws.gov/nwi/plants.htm.1988.

[35] Kathryn M. Flinn, Martin J. Lechowicz, and Marcia J. Waterway Plant Species Diversity And Composition Of Wetlands Within An Upland Forest American Journal of Botany 95(10): 1216-1224.2008. 